\title{
HIV-associated sensory polyneuropathy and neuronal injury are associated with miRNA-455-3p induction
}

\author{
Eugene L. Asahchop, ${ }^{1}$ William G. Branton, ${ }^{1}$ Anand Krishnan, ${ }^{1}$ Patricia A. Chen, ${ }^{1}$ Dong Yang, ${ }^{2}$ \\ Linglong Kong, ${ }^{2}$ Douglas W. Zochodne, ${ }^{1,3}$ Bruce J. Brew, ${ }^{4}$ M. John Gill, ${ }^{5}$ and Christopher Power ${ }^{1,3,5}$ \\ 'Department of Medicine (Neurology), University of Alberta, Edmonton, Alberta, Canada. ${ }^{2}$ Department of Mathematical \\ and Statistical Sciences, University of Alberta, Edmonton, Alberta, Canada. ${ }^{3}$ Neuroscience and Mental Health Institute, \\ University of Alberta, Edmonton, Alberta, Canada. ${ }^{4}$ Departments of Neurology and HIV, St. Vincent's Hospital, and Peter \\ Duncan Neurosciences Unit, St. Vincent's Centre for Applied Medical Research, University of New South Wales, Sydney, \\ Australia. ${ }^{5}$ Department of Medicine, University of Calgary, Calgary, Alberta, Canada.
}

\begin{abstract}
Symptomatic distal sensory polyneuropathy (SDSP) is common and debilitating in people with HIV/AIDS, leading to neuropathic pain, although the condition's cause is unknown. To investigate biomarkers and associated pathogenic mechanisms for SDSP, we examined plasma miRNA profiles in HIV/AIDS patients with SDSP or without SDSP in 2 independent cohorts together with assessing related pathogenic effects. Several miRNAs were found to be increased in the Discovery Cohort (sDSP, $n=29$; non-DSP, $n=40$ ) by array analyses and were increased in patients with sDSP compared with patients without sDSP. miR-455-3p displayed a 12-fold median increase in the SDSP group, which was confirmed by machine learning analyses and verified by reverse transcription PCR. In the Validation Cohort $(\operatorname{sDSP} n=16$, non-DSP $n=20$, healthy controls $n=$ 15), significant upregulation of miR-455-3p was also observed in the SDSP group. Bioinformatics revealed that $\mathrm{miR}-455-3 p$ targeted multiple host genes implicated in peripheral nerve maintenance, including nerve growth factor (NGF) and related genes. Transfection of cultured human dorsal root ganglia with miR-455-3p showed a concentration-dependent reduction in neuronal $\beta$-III tubulin expression. Human neurons transfected with miR-455-3p demonstrated reduced neurite outgrowth and NGF expression that was reversed by anti-miR-455-3p antagomir cotreatment. miR-455-3p represents a potential biomarker for HIV-associated sDSP and might also exert pathogenic effects leading to sDSP.
\end{abstract}

Conflict of interest: MJG has served as an ad hoc member of national HIV advisory boards to Merck, Gilead, and Viiv Healthcare in the last 3 years.

License: Copyright 2018, American Society for Clinical Investigation.

Submitted: May 29, 2018

Accepted: October 24, 2018

Published: December 6, 2018

Reference information: JCI Insight. 2018;3(23):e122450. https://doi.org/10.1172/jici. insight.122450.

\section{Introduction}

Chronic pain is the most common neurological complication observed in people with HIV/AIDS (> 40\%) and is associated with increasing age, female sex, greater comorbidity, and neuropsychiatric disease in the setting of active care including antiretroviral therapy (ART) (1-3). Symptomatic distal sensory polyneuropathy (sDSP) is frequently encountered as a neuropathic pain syndrome associated with HIV-1 infection, particularly with sustained immunosuppression or exposure to older ART drugs, such as stavudine, didanosine, and zalcitabine $(4,5)$. Nonetheless, sDSP remains a contemporary challenge in patients receiving nontoxic ART that affects over $20 \%$ of HIV/AIDS patients in active care (6-8). Typical symptoms include a persistent burning sensation in the feet, complicated by allodynia, hyperalgesia, numbness, and paresthesia that are more common in taller and older patients. Clinical signs comprise reduced sensation that is more pronounced in the feet than the hands, absent or reduced deep tendon reflexes (especially at the ankles), and an antalgic gait necessitating, if sufficiently severe, the use of a wheelchair. Treatments for sDSP are limited to symptomatic relief using anticonvulsants (9), topical capsaicin (10), smoked cannabis (11), acupuncture (12), and opiates in select patients (13). In one study recombinant human nerve growth factor (NGF) delivered locally improved pain symptomatology but did not enhance nerve recovery (14). Numerous reports point to the pathogenic effects of inflammatory molecules and individual HIV-1 proteins (e.g., Vpr, gp120) on peripheral nerves, dorsal root ganglia, and in the CNS in the pathogenesis of SDSP (15), but the precise molecular mechanisms underpinning neural cell injury in SDSP remain uncertain. Although sDSP is characterized by substantial 
Table 1. Sociodemographic and clinical features of HIV/AIDS patients with or without SDSP in the Discovery Cohort ${ }^{A}$

\begin{tabular}{|c|c|c|c|c|}
\hline & Non-DSP $(n=40)$ & $\operatorname{sDSP}(n=29)$ & Controls $\left(\right.$ HIV $\left.^{-}\right)(n=9)$ & $P$ value $^{\mathrm{B}}$ \\
\hline Age (years) & $41.35 \pm 9.56$ & $45.21 \pm 11.90$ & $33.78 \pm 9.24$ & NS \\
\hline Sex (male) & $77.50 \%$ & $82.76 \%$ & $66.67 \%$ & NS \\
\hline Duration of HIV (years) & $6.99 \pm 6.87$ & $6.91 \pm 7.24$ & NA & NS \\
\hline HAND & $40 \%$ & $55.17 \%$ & NA & NS \\
\hline Recent viral load median (log copies/ml) & 2.819 & 1.799 & NA & NS \\
\hline AIDS (nadir CD4 T cell count $<200$ count $/ \mathrm{mm}^{3}$ ) & $57.50 \%$ & $68.97 \%$ & NA & 0.0135 \\
\hline CD8 T cell (log count $\left./ \mathrm{mm}^{3}\right)$ & $2.89 \pm 0.17$ & $2.92 \pm 0.29$ & NA & NS \\
\hline HCV seropositivity & $10 \%$ & $13.79 \%$ & NA & NS \\
\hline Diabetes & $7.50 \%$ & $13.79 \%$ & NA & NS \\
\hline Syphilis & $17.50 \%$ & $17.20 \%$ & NA & NS \\
\hline
\end{tabular}

${ }^{A}$ Mean \pm SD or percentage. ${ }^{B} N S$, not significant. HAND: HIV-associated neurocognitive disorder; HCV: hepatitis C virus.

comorbidity, reduced quality of life, and increased health care costs, there is limited knowledge regarding its pathogenesis, potential biomarkers for diagnosis, or options for disease-modifying therapy (16).

miRNAs are noncoding RNA molecules of which the active forms (22 nucleotides) are derived from processing larger nuclear DNA-encoded pre-miRNAs by specific enzymes (17). These miRNAs exert their effects through their complementarity to the $3^{\prime}$ end of specific host genes to cause suppression of protein translation by ribosomes, destabilization of polyA tails on RNA molecules, and facilitation of RNA transcript cleavage, although other mechanisms underlying miRNAs' actions have been proposed (18). When present in exosomes, miRNAs are stable and can exert sustained effects in different tissues, including the nervous system (19). Furthermore, they are released into the circulation and have been investigated as potential biomarkers for the diagnosis of different diseases. Substantial evidence exists suggesting HIV/AIDS pathogenesis is modulated by miRNAs (20-22). Recently, the contribution of miRNAs to diabetic neuropathy as well as other painful peripheral neuropathies has been shown in both clinical and experimental studies $(20,23)$.

Our working hypothesis was that differentially expressed miRNAs predicted the clinical occurrence of sDSP as well as mediated the underlying disease mechanisms contributing to sDSP. Using 2 clinical cohorts and several experimental platforms, we investigated this hypothesis and found that miR-455-3p in plasma from patients with HIV/AIDS with sDSP was induced by and indicative of sDSP. The findings also suggested that $\mathrm{miR}-455-3 \mathrm{p}$ promoted dorsal root neuronal injury.

\section{Results}

Clinical cohorts. The Discovery Cohort (Table 1) was composed of HIV/AIDS patients assessed and diagnosed with sDSP or non-DSP at the Southern Alberta Clinic (SAC). The Validation Cohort (Table 2) was derived from a cohort at St. Vincent's Hospital diagnosed with either sDSP or non-DSP. Patients with sDSP or non-DSP in the Discovery Cohort were matched in terms of mean age, sex, education level, estimated duration of HIV-1 infection, and ethnicity as well as the prevalence of HAND, HCV, syphilis, and diabetes but differed significantly in nadir $\mathrm{CD}^{+} \mathrm{T}$ cell counts (Table 1 ). In the Validation Cohort, the sDSP and non-DSP groups did not differ with respect to all demographic or clinical variables (Table 2). For both cohorts, healthy controls (HCs) were recruited with informed consent at each site.

Differentially expressed miRNAs in sDSP. Plasma miRNA profiles were analyzed and compared in the Discovery Cohort using GeneSpring analyses and subjected to statistical assessment. Hierarchical clustering analysis showed distinct miRNA profiles. The revealed profiles indicated that multiple miRNAs were upregulated or downregulated in the SDSP compared with the non-DSP group (Figure 1A). Comparative analyses identified several miRNAs that displayed significantly increased expression in the sDSP group compared with the non-DSP group (a fold change $\geq 2.0$ with associated $P<0.05$ ) (Figure $1 \mathrm{~B}$ ). Among these, miR-455-3p showed a 12-fold increase in the sDSP group compared with the non-DSP group while other miRNAs displayed significant increases in expression. Upon correcting for multiple comparisons 
Table 2. Sociodemographic and clinical features of HIV/AIDS patients with or without sDSP in the Validation Cohort ${ }^{A}$

\begin{tabular}{|c|c|c|c|c|}
\hline & nonDSP $(n=16)$ & $\operatorname{sDSP}(n=24)$ & Controls $\left(\mathrm{HIV}^{-}\right)(n=19)$ & $P$ value ${ }^{B}$ \\
\hline Sex (male) & $91 \%$ & $75 \%$ & $57 \%$ & NS \\
\hline Recent viral load (log copies/ml) & $1.8 \pm 1.3$ & $1.8 \pm 1.2$ & NA & NS \\
\hline Recent CD4 T cell (count/mm³) & $398.2 \pm 2.3$ & $316.2 \pm 2.0$ & NA & NS \\
\hline CD4 T cell nadir (count/mm³) & $125.9 \pm 5.6$ & $125.9 \pm 3.6$ & NA & NS \\
\hline
\end{tabular}

(with Bonferroni) among the identified miRNAs, we found that a single miRNA, miR-455-3p, passed the correction test for multiple comparisons. miR-455-3p and miR-940 (one of the miRNAs with a high fold change although it did not pass the Bonferroni correction test) were selected among the upregulated miRNAs for further analysis. Based on array expression data, the receiver operating characteristic (ROC) curves for both miRNAs were predictive for sDSP: miR-455-3p, AUC 0.83, and miR-940, AUC 0.74 (Supplemental Figure 1, A and B; supplemental material available online with this article; https://doi.org/10.1172/ jci.insight.122450DS1). We also performed correlational analyses of these 2 miRNAs' expression levels with several relevant clinical variables. There were significant correlations between miR-455-3p levels and plasma viral load $(r=0.3), \mathrm{CD}^{+} \mathrm{T}$-cell $(r=0.3)$ counts in blood, and current CD4 ${ }^{+} \mathrm{T}$-cell $(r=-0.3)$ counts in blood (Supplemental Figure 1, C-F). In contrast, miR-940 was correlated significantly with plasma viral load only $(r=0.3)$. Several other miRNAs were downregulated at this cutoff level, but none passed the Bonferroni correction test. We therefore did not consider any downregulated miRNAs for further analysis.

To confirm the above analyses, we applied machine learning approaches to the data. Screening the data using $P$ value ranking before applying machine learning did not show differences from the unscreened data. The bars indicated the selection frequency of the top-ranking miRNAs in 1,000 and 100 runs for Random Forest Analysis (Figure 1C) and for Support Vector Machines (Supplemental Figure 2), respectively. Although the frequencies of miRNAs showed different patterns in the 2 approaches, miR-455-3p, which has a frequency of 932 in Random Forest Analysis and 100 in Support Vector Machines, displayed a dominant effect in both approaches. This implied that the expression levels of miR-455-3p classified patients into the sDSP or non-DSP groups. In the Support Vector Machines approach, the dominant effect of miR455-3p was especially evident, with the second-ranking gene, miR-4706, showing a frequency of only 41.

Quantitative PCR verification studies. Given the predominance of miR-455-3p and the need to confirm the above observations, a quantitative PCR (qPCR) assay was used to measure the expression levels of $\mathrm{miR}-455-3 \mathrm{p}$ as well as miR-940, relative to $\mathrm{miR}-16-5 \mathrm{p}$, in plasma samples from the Discovery Cohort. miR-455-3p and miR-940 were consistently amplified in all samples, unlike other miRNAs that showed induction in the above array analyses (Figure 1). The expression levels of these 2 miRNAs were examined, revealing that both miR-940 (Figure 2A) and miR-455-3p (Figure 2B) were increased in the sDSP group compared with the non-DSP and HC groups. Based on the qPCR analyses, ROC curves for both miRNAs showed high predictive values for sDSP: miR-940, AUC 0.85 (Figure 2C), and miR-455-3p, AUC 0.82 (Figure 2D), and which resembled the ROC curves derived from the miRNA array data (Supplemental Figure 1, A and B). These data prompted examination of an independent cohort with and without sDSP.

Investigation of $\mathrm{miR}-455-\mathrm{p} 3$ and miR-940 in plasma samples from the Validation Cohort disclosed that miR-455-3p was significantly increased in the sDSP group (Figure 3A), which was reflected in the corresponding ROC curve, AUC 0.73 (Figure 3C). In contrast, miR-940 was not increased in sDSP (Figure 3B), which was also apparent in the matched ROC curve, AUC 0.61 (Figure 3D). Collectively, these observations pointed to $\mathrm{miR}-455-3 \mathrm{p}$ as representing a consistently upregulated miRNA, whereas miR-940 was induced only in the Discovery Cohort, in plasma from HIV/AIDS patients with sDSP.

Genes targeted by individual miRNAs. miRNAs regulate gene function by base pairing of the miRNA with the host mRNA, usually at the $3^{\prime}$ UTR, to regulate the expression of the targeted gene. Using miRwalk, several mRNA targets were predicted for miR-455-p3 and miR-940 and confirmed by other algorithms. To investigate this list in more depth and select targets with a high probability of miRNA-mRNA interaction, we searched for targets that were predicted by both $\mathrm{miR}-455-3 p$ and miR-940 in at least 2 algorithms for 
A

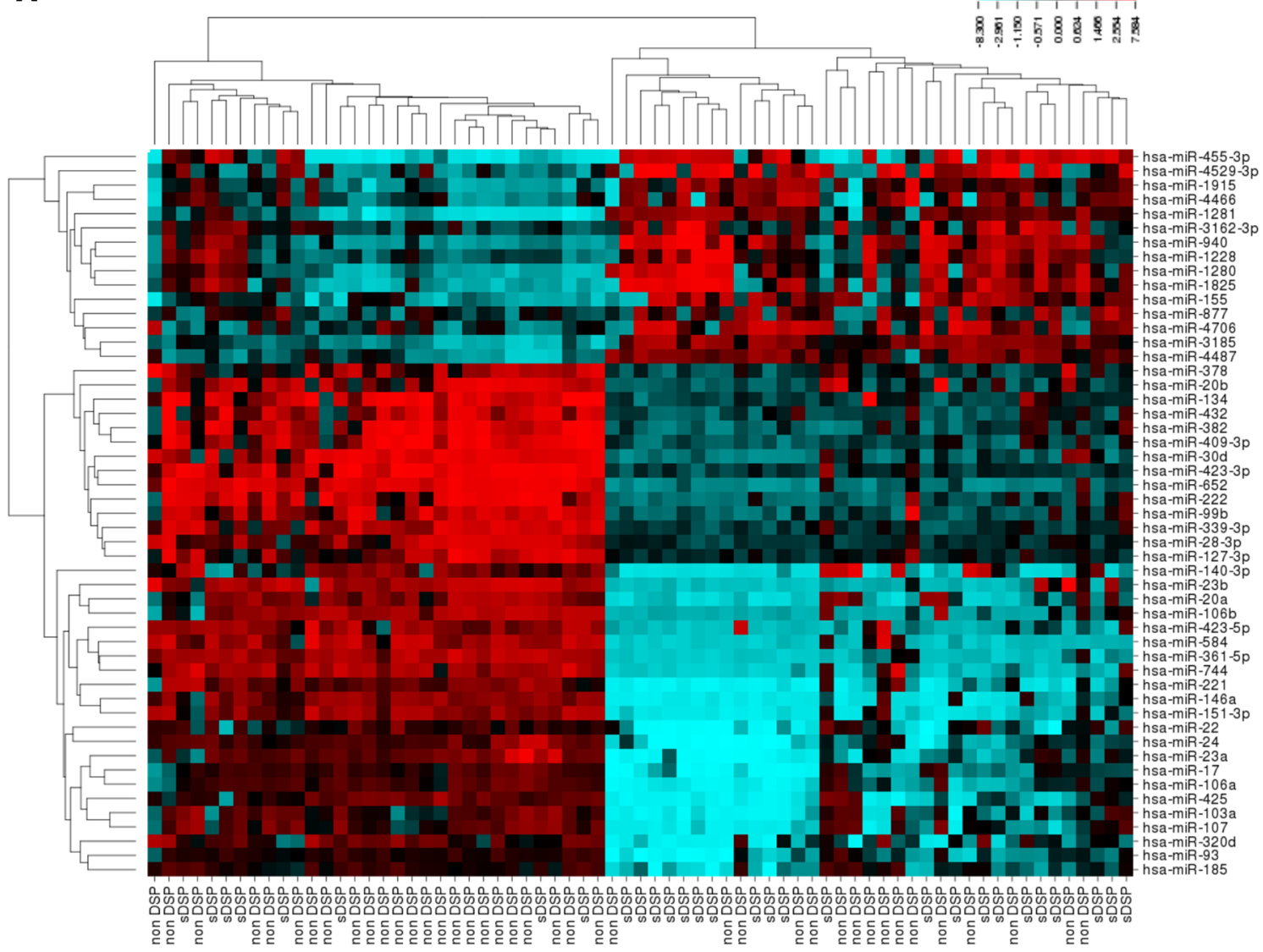

B

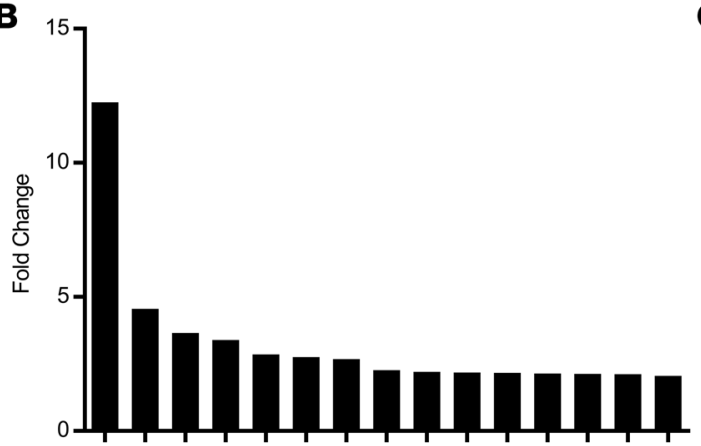

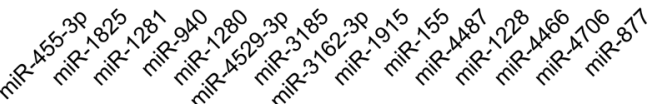

C

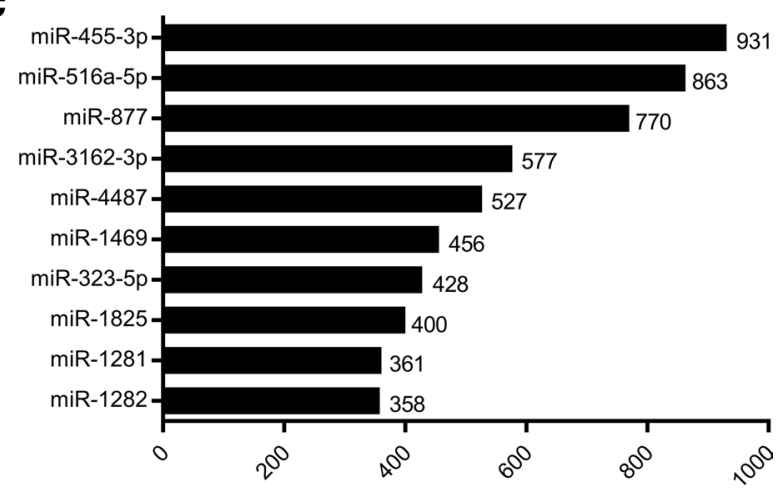

Frequency of occurence of miRNA

Figure 1. Differentially expressed miRNAs in the Discovery Cohort. Analysis of upregulated and downregulated miRNAs analyzed by GeneSpring analyses in sDSP $(n=29)$ compared with non-DSP $(n=40)$ patients in the Discovery Cohort. (A) Hierarchical cluster. The heat map clusters and distinguishes the upregulated (top 15 miRNAs) and downregulated (bottom 36 miRNAs) miRNA profile substantially and as shown by the grouping and clustering of the side dendrograms (left). The heat map also clusters the patients into SDSP and non-DSP, as shown by the 2 groups in the top dendrogram. (B) Histogram of upregulated miRNAs. The median of each probe set in the sDSP or non-DSP patients was calculated and the significance of any differences determined by Mann-Whitney $U$ test. The 15 upregulated miRNAs with a fold change of greater than or equal to 2 and a $P$ value less than or equal to 0.05 in the array hybridization were selected. (C) Random Forest Analysis shows the top 10 miRNAs with the highest frequency of miRNA occurrence based on $P$ value $(P<0.05)$ and 2-tailed sample $t$ test.

each of the miRNAs. All target genes (Table 3) were predicted for both miR-455-3p and miR-940, apart from $N G F$, which was predicted by only miR-455-3p. Interestingly, $N G F$ was predicted by 4 algorithms (DIANA-microT v3.0, miRanda, miRwalk, and PICTAR5). Furthermore, NGFR (a receptor for NGF, P75NTR) was predicted as a target gene for both miR-455-3p and miR-940 (Table 3). Of note, it has been shown previously that NGF was linked to HIV-associated sDSP (24). In summary, these bioinformatic 
A

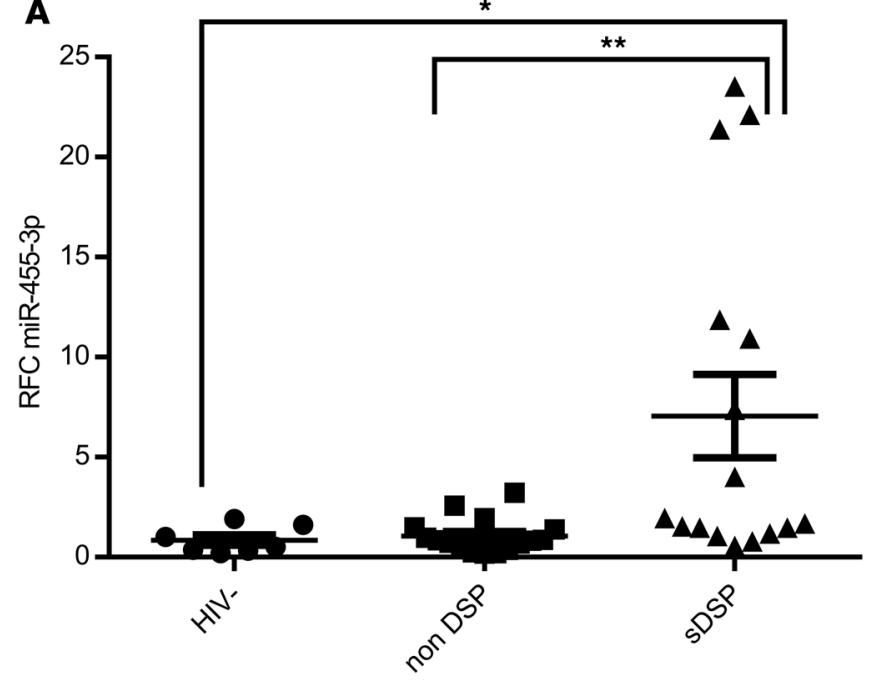

C

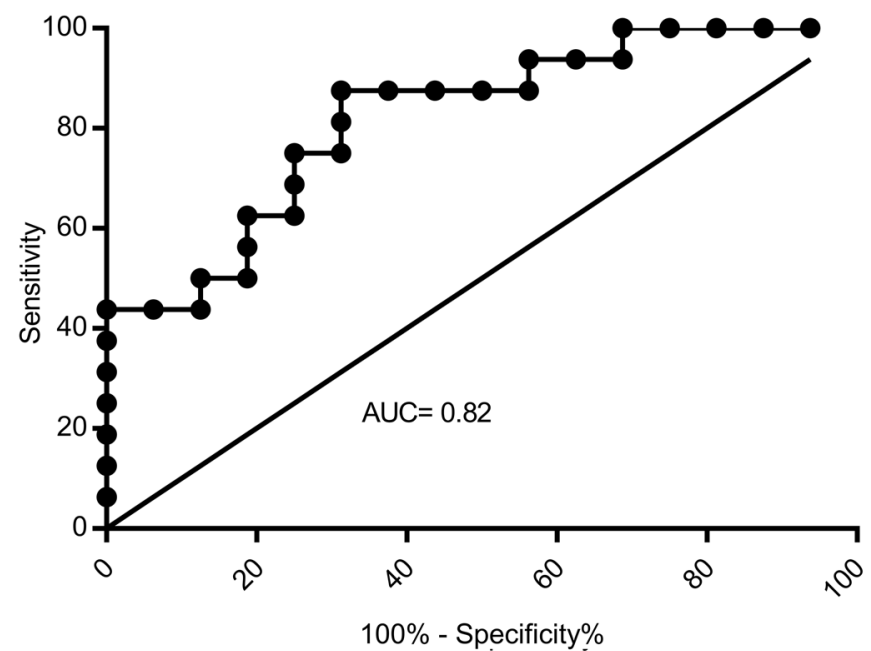

B

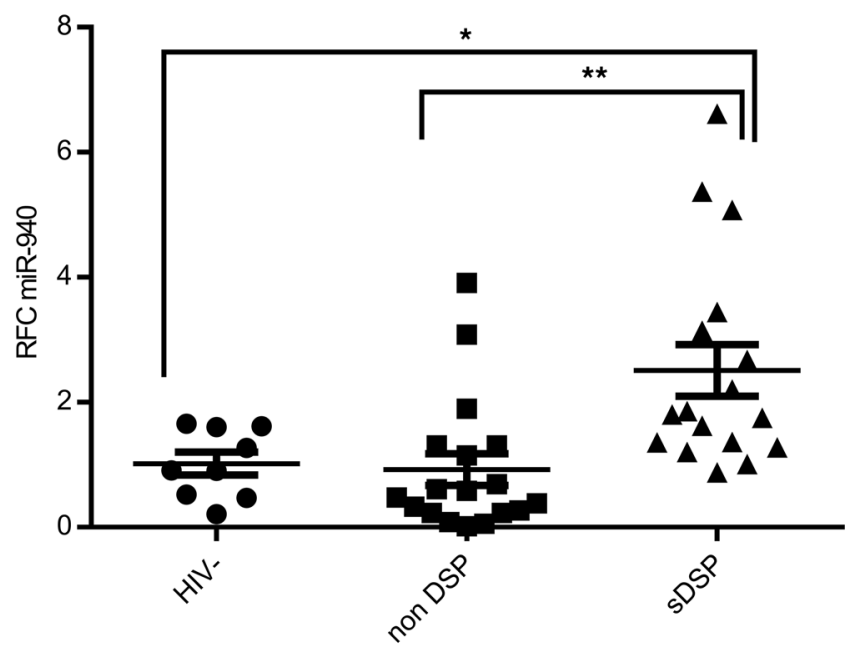

D

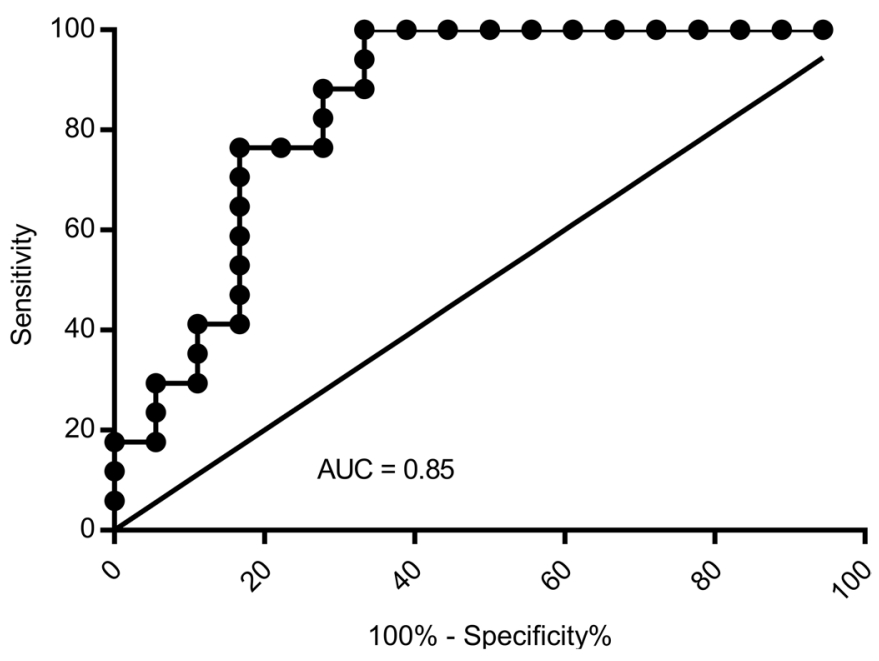

Figure 2. miRNA expression and prediction in SDSP by qPCR in the Discovery Cohort. (A) The miR-940 and (B) the miR-455-3p expression profiles were quantified in the plasma of sDSP $(n=16)$, non-DSP $(n=16)$, and HC $(n=9)$ patients in the Discovery Cohort by qPCR assay to verify the array hybridization results. MiRNA expression was normalized to miR-16-5p expression. The results were analyzed using 1-way ANOVA (Kruskal-Wallis) test. The ROC curve analysis (C) for miR-940 shows an AUC of 0.85 and (D) for miR-455-3p shows an AUC of $0.82 .{ }^{*} P<0.05,{ }^{* *} P<0.01$. RFC: relative fold change; NS: not significant. Horizontal bars indicate the mean \pm SD.

analyses pointed to multiple genes targeted by miR-455-3p that were related to neural cell functions implicated in the pathogenesis of sDSP. Thus, we examined the interactions between miR-455-3p and neural cell viability, particularly $N G F$ expression, that might explain the involvement of miR-455-3p in sDSP.

miRNA activation in neural cells. Because miR-455-3p showed upregulation in plasma from HIV/AIDS patients with sDSP and the source of this miRNA was unknown, the capacity of the neurotoxic HIV-1 protein, viral protein $\mathrm{R}(\mathrm{Vpr})$, to induce miRNAs was examined because of its reported contribution to the development of sDSP (24). Vpr (100 nM) was applied for 48 hours to human neuronal cells (SK-N-SH), which resulted in reduced $\beta$-III tubulin immunoreactivity in cultured neurons (Supplemental Figure 3A), although overall cellular viability, as measured by DAPI labeling, was unaffected (Supplemental Figure 3B). Vpr exposure also caused a significant increase of miR-455-3p expression in cells (Supplemental Figure 3C), but miR-940 expression was not significantly increased (Supplemental Figure 3D). These observations indicated that an established neurotoxic HIV-1 protein induced miR-455-3p in neural cells without killing cells despite altering their structural protein expression. 
A

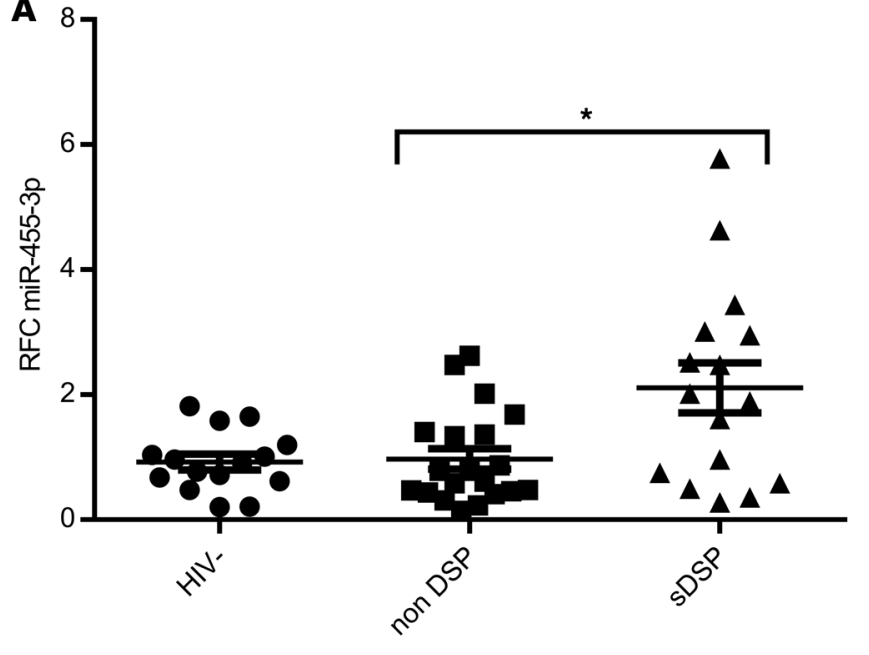

C

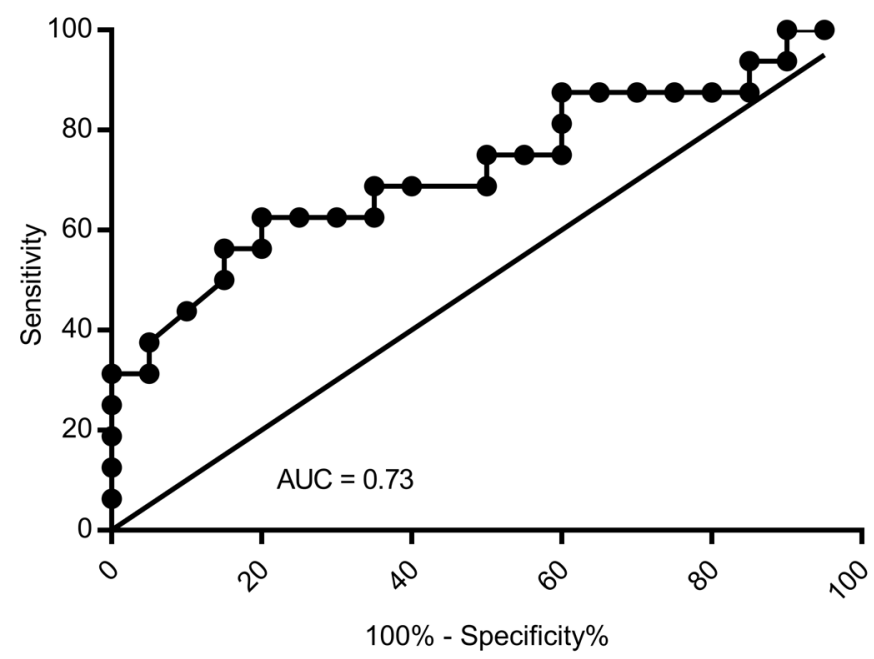

B

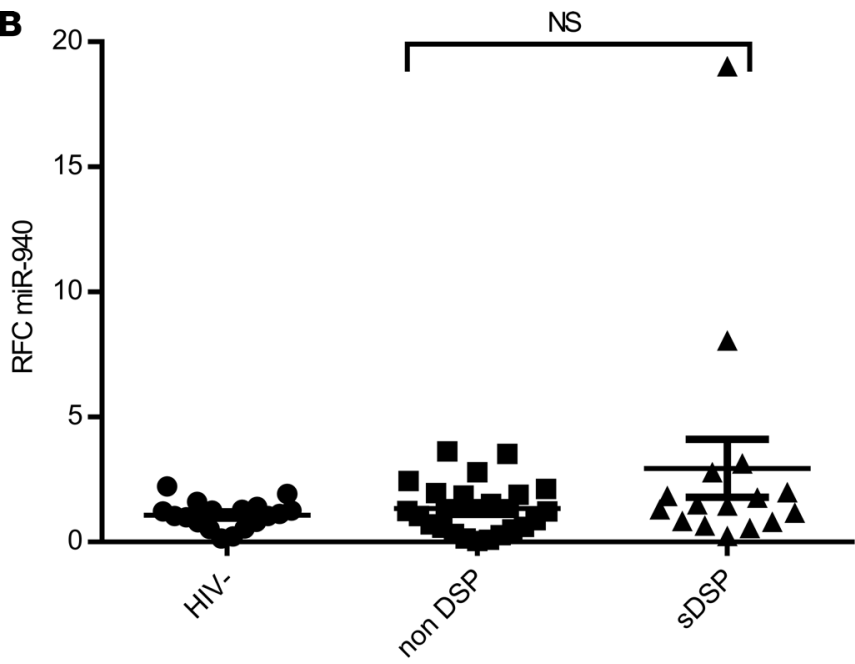

D

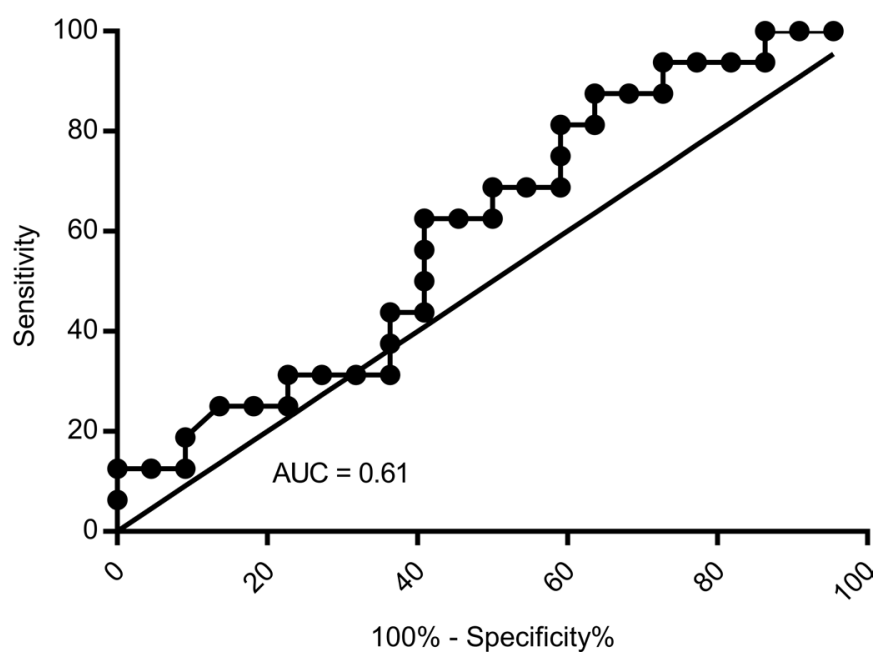

Figure 3. miRNA expression and prediction in SDSP by qPCR in the Validation Cohort. (A) The miR-455-3p and (B) the miR-940 expression profiles were quantified in the plasma of $\operatorname{sDSP}(n=16)$, non-DSP $(n=20)$, and HC $(n=15)$ patients in the Validation Cohort by qPCR assay. MiRNA expression was normalized to miR-16-5p expression (that was observed to be constitutively expressed the same in SDSP and non-DSP patients). The results were analyzed using 1-way ANOVA (Kruskal-Wallis) test. The ROC curve analysis (C) for miR-455-3p shows an AUC of 0.73 and (D) for miR-940 shows an AUC of 0.61. ${ }^{*} P<0.05,{ }^{* *} P<0.01$, NS: not significant. Horizontal bars indicate the mean \pm SD.

Effects of miR-455-3p in human dorsal root ganglia. Because miR-455-3p was induced in neural cells by an HIV-1 protein and targeted several genes implicated in neuronal maintenance and growth, we transfected cultured fetal human dorsal root ganglia (hDRGs) with this miRNA. hDRGs transfected with noncoding miRNA (miRNA-NC) exhibited $\beta$-III tubulin in neuronal processes (Figure 4A) and NGF in immunoreactivity within neuronal soma (Figure 4B). In contrast, hDRGs transfected with miR-455-3p showed reduced $\beta$-III tubulin (Figure 4D) and NGF (Figure 4E) immunoreactivity in a dose dependent manner (Figure $4 \mathrm{~K}$ ), whereas cotransfection of $\mathrm{miR}-455-3 \mathrm{p}$ and its antagomir (inhibitor) did not affect $\beta$-III tubulin (Figure 4G) expression levels. Moreover, miR-455-3p transfection displayed a concentration-dependent reduction in $\beta$-III tubulin expression compared with human dorsal root ganglion (hDRG) cultures transfected with miRNA-NC (Figure 4J). These observations were confirmed by showing that cotransfection with the antagomir complementary to $\mathrm{miR}-455-3 \mathrm{p}$ as well as $\mathrm{miR}-455-3 \mathrm{p}$ at the same time rescued suppression of $\beta$-III tubulin immunoreactivity (Figure $4 \mathrm{H}$ ) by miRNA-455-3p (Figure $4 \mathrm{~K}$ ). Nonetheless, the trend toward reduced NGF expression in hDRGs following miR-455-3p transfection was nonsignificant (Supplemental Figure 4A). In summary, miR-455-3p exerted inhibitory effects on neuronal process formation that was prevented by coapplication of its antagomir. 
Table 3. Predicted mRNA target genes for miR-455-3p and miR-940

\begin{tabular}{|c|c|c|c|c|}
\hline \multirow{2}{*}{ Gene ID } & \multirow{2}{*}{ Gene name } & \multirow{2}{*}{ Function } & \multicolumn{2}{|c|}{ Number of algorithms used } \\
\hline & & & $\operatorname{miR}-455-3 p$ & miR-940 \\
\hline NGF & Nerve growth factor & Neurotrophic factor & 4 & 0 \\
\hline NGFR (P75NTR) & Nerve growth factor receptor & Receptor for NGF & 5 & 2 \\
\hline NF2 & Neurofibromin 2 & Regulation of ion transport & 3 & 5 \\
\hline IL-16 & IL-16 & modulator of $\mathrm{T}$ cell activation & 5 & 3 \\
\hline TNFAIP2 & $\begin{array}{l}\text { TNF- } \alpha \text { induced } \\
\text { Protein } 2\end{array}$ & Inflammation & 3 & 5 \\
\hline IL-10RA & IL-10 receptor subunit- $\alpha$ & $\begin{array}{l}\text { inhibits the synthesis of } \\
\text { proinflammatory cytokines }\end{array}$ & 3 & 5 \\
\hline ICAM1 & Intercellular adhesion molecule 1 & $\begin{array}{c}\text { Promotes the assembly of endothelial } \\
\text { apical }\end{array}$ & 6 & 5 \\
\hline IGF1 & Insulin like growth factor 1 & Growth and development & 4 & 4 \\
\hline CD4 & CD4 molecule & $\begin{array}{l}\text { Function as an important mediator of } \\
\text { indirect neuronal damage }\end{array}$ & 2 & 4 \\
\hline APOBECЗF & $\begin{array}{l}\text { Apolipoprotein B editing enzyme catalytic } \\
\text { polypeptide-like 3F }\end{array}$ & Antiviral activity & 4 & 6 \\
\hline MAP2K7 & Mitogen-activated protein kinase kinase 7 & $\begin{array}{l}\text { Mediate the cell responses to } \\
\text { proinflammatory cytokines }\end{array}$ & 3 & 5 \\
\hline NTRK2 (Trk-B) & Neurotrophic receptor tyrosine kinase 2 & $\begin{array}{c}\text { Development and the maturation of } \\
\text { the central and the peripheral nervous } \\
\text { systems through regulation of neuron } \\
\text { survival, proliferation, migration }\end{array}$ & 2 & 3 \\
\hline NFASC & Neurofascin & $\begin{array}{c}\text { Neurite outgrowth, neurite fasciculation, } \\
\text { and organization of the axon initial } \\
\text { segment and nodes of Ranvier on axons }\end{array}$ & 5 & 2 \\
\hline
\end{tabular}

miR-455-3p suppresses neurite outgrowth of human neurons. Because miR-455-3p transfection resulted in suppression of $\beta$-III tubulin expression in hDRGs, this action was explored further in differentiated human neuronal (SK-N-SH) cells because of the monoclonality of these cells. To confirm the efficiency of transfection, we verified miR-455-3p expression in transfected cells by qPCR (Supplemental Figure 4B). SK-N-SH cells transfected with an miRNA-NC displayed intense $\beta$-III tubulin (Figure 5A) and NGF (Figure 5B) immunoreactivity compared with cells transfected with miR-455-3p (Figure 5, D and E). Similarly, miR-455-3p attenuated analysis of neurite outgrowth by reducing the average neuronal process length of outgrowth (Supplemental Figure 4C). Indeed, miR-455-3p significantly reduced $\beta$-III tubulin expression in neuronal cells relative to miRNA-NC, which was rescued by cotransduction with the antagomir (Figure 5G). NGF expression was significantly suppressed by miR-455-3p compared with miRNA-NC, but the antagomir again restored the reduced NGF expression level (Figure 5H). Thus, miR-455-3p transfection suppressed NGF expression that was rescued by a specific antagomir.

\section{Discussion}

This report documents the first description, to our knowledge, of an miRNA associated with sDSP in patients with HIV/AIDS. The miRNA (miR-455-3p) was significantly increased in plasma from 2 independent cohorts with ROC analyses that were predictive of sDSP. Moreover, miR-455-3p targeted several genes directly implicated in the pathogenesis (and treatment) of sDSP, including nerve growth factor (NGF), based on multiple bioinformatic algorithms. Indeed, we verified this observation in an ex vivo transfection study showing that miR-455-3p mediated a reduction of NGF expression and accompanying reduced neuronal processes that were reversed by an anti-miR-455-3p antagomir. Thus, these observations highlight a new potential biomarker for SDSP while pointing to a novel molecular mechanism (to our knowledge) by which sDSP occurs in patients with HIV/AIDS.

SDSP remains a clinical challenge affecting at least $20 \%$ of patients with HIV/AIDS receiving contemporary care, especially in older age and taller female patients $(25,26)$, for which the diagnostic guidelines remain largely clinically based without definitive biomarkers. Antisulfatide antibodies as 

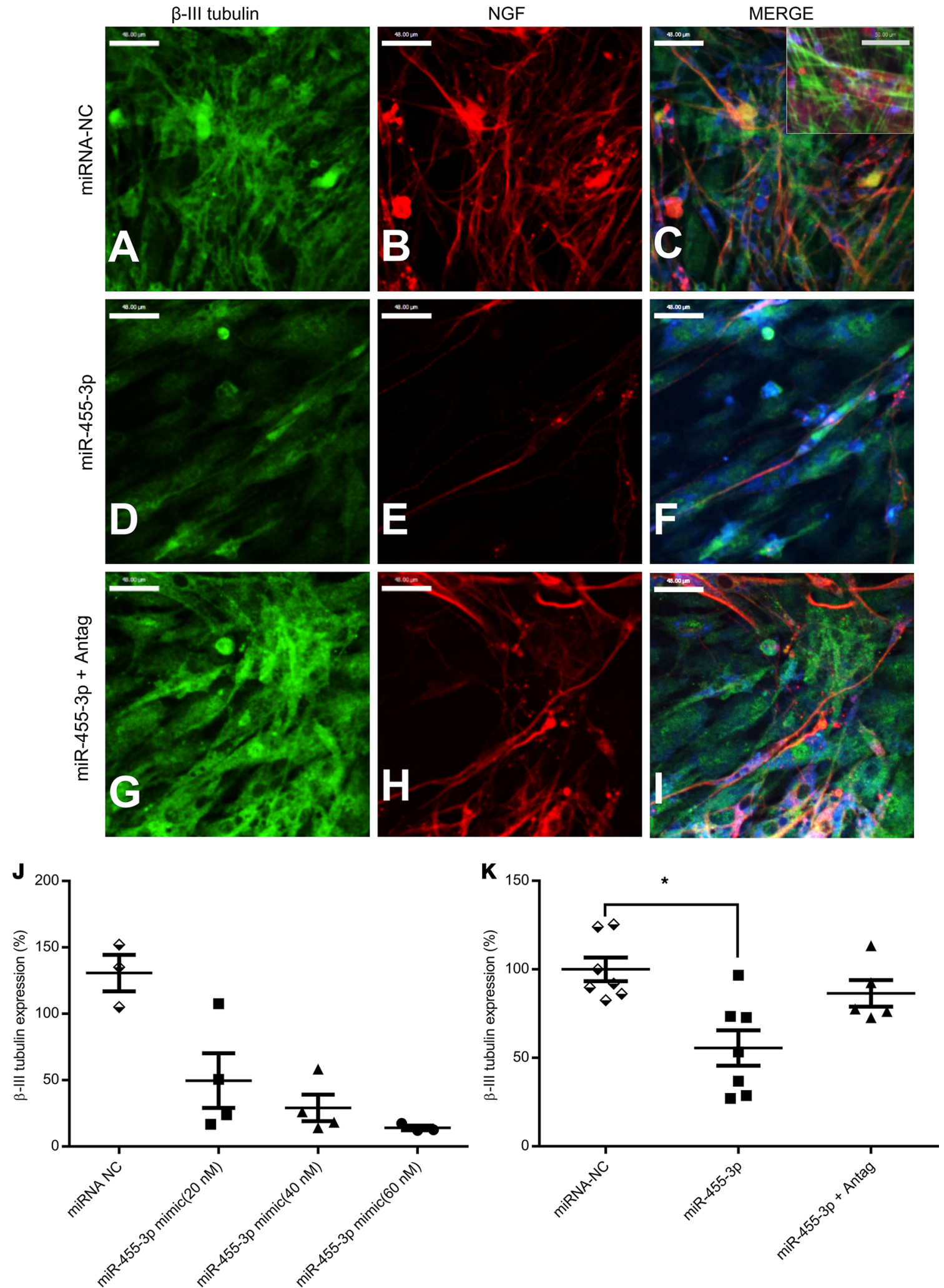

Figure 4. Immunofluorescence and immunodetection in hDRGs. Cultured hDRGs were transfected with (A-C) miRNA-NC, (D-F) miR-455-3p, or (G-I) miR455-3p and antagomir for 48 hours. After transfection, cells were fixed and immunolabeled with anti- $\beta$-III tubulin (green) or anti-NGF (red) or stained with DAPI (blue). Images were captured by confocal microscopy. Immunofluorescence was a single experiment in triplicates or quadruplets. Scale bars 48 $\mu$ m. (J) For in-cell immunodetection, cultured hDRGs were transfected with miRNA-NC or miR-455-3p at different concentrations for 48 hours. This experiment was repeated 3 times. This shows a representative experiment. (K) Cultured hDRGs were transfected with miRNA-NC, miR-455-3p, or miR-455-3p and antagomir for 48 hours. After transfection, cells were incubated with anti- $\beta$-III tubulin antibodies and immunofluorescence was quantified. In-cell analysis experiments were done 3 times and the results merged, averaged, and analyzed using 1-way ANOVA (Kruskal-Wallis) test. ${ }^{*} P<0.05$, ${ }^{*} P<0.01$. Antag, antagomir. 

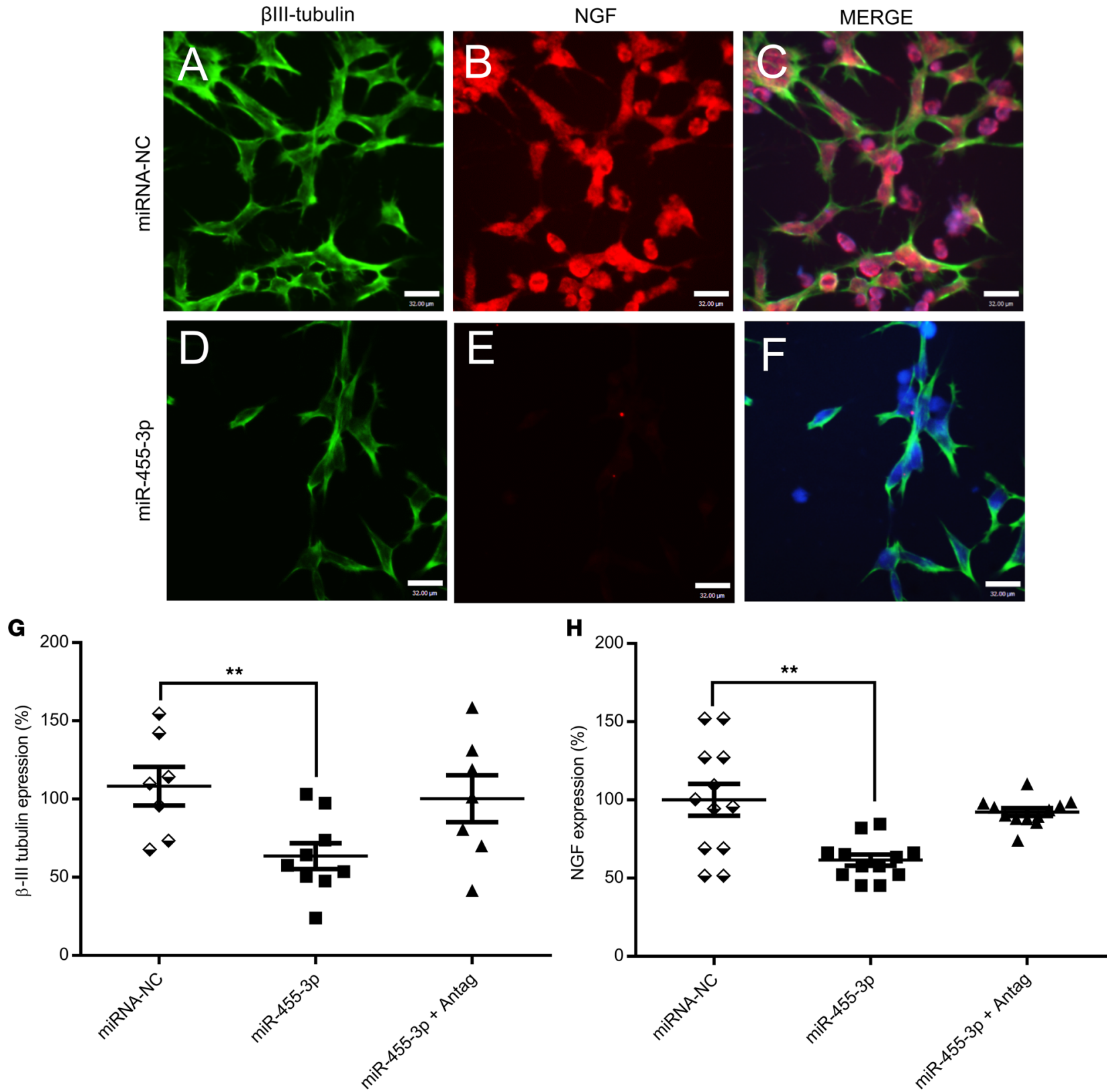

Figure 5. Immunofluorescence and in-cell immunodetection in human neurons. SK-N-SH cells were differentiated for 48 hours followed by transfection with (A-C) miRNA-NC or (D-F) miR-455-3p for 48 hours. Scale bars $32 \mu \mathrm{m}$. After transfection, cells were fixed and immunolabeled with anti- $\beta$-III tubulin (green) or anti-NGF (red) antibodies or stained with DAPI (blue). Immunofluorescence was a single experiment in triplicates or quadruplets. For in-cell Western analyses, differentiated SK-N-SH cells were transfected with miRNA-NC, miR-455-3p, or miR-455-3p with an miR-455-3p-specific antagomir for 48 hours. After transfection, cells were fixed, incubated with (C) anti- $\beta$-III tubulin or (H) anti-NGF antibodies, and quantified by in-cell Western analysis. All in-cell Western analysis experiments were done 3 times and the results merged, averaged, and analyzed using 1-way ANOVA (Kruskal-Wallis) test. $\left({ }^{*} P<0.05,{ }^{* *} P<0.01\right)$. Horizontal bars indicate the mean \pm SD.

well as mitochondrial gene mutations have been associated with $\operatorname{sDSP}(27,28)$, and specific HIV-1 proteins, including Vpr and gp120 (22, 24, 29), are reported to contribute through uncertain mechanisms to sDSP. The underlying pathogenesis is complex, likely involving selective injury to hDRG neurons causing retraction of small-diameter sensory fibers that is evident in skin biopsies from patients with sDSP (30). There is also likely remodeling of afferent inputs to the spinal cord at the level of the dorsal horn. The diffuse microglial activation throughout the spinal cord (and brain) that occurs during HIV/ AIDS might also contribute to the development of neuropathic pain and altered sensory processing (31, 32). In fact, our findings indicate that upregulation of miR-455-3p could be due to HIV-1 Vpr's actions 
on neurons and not resulting from antiretroviral toxic neuropathy. These complex pathogenic circumstances are further influenced by exposure to substances of abuse that can modulate pain perception thresholds $(33,34)$. A reliable clinical biomarker for sDSP that can be used for diagnosing sDSP as well as ascertaining potential responses to therapies would be of immense value.

Analyses of tissue levels of potential biomarkers for sDSP and other neuropathies face substantial challenges because of the difficulty in obtaining biopsied samples, which in SDSP is limited to skin biopsies harvested for epidermal axonal counts $(35,36)$. Thus, plasma-derived molecules represent an attractive alternative approach in searching for disease biomarkers. Plasma miRNAs are comparatively stable and abundant, permitting implementation of array techniques for discovering specific biomarkers, and are also amenable to validation by other methods in large and separate cohorts, as described herein. Although multiple miRNAs showed increased expression in the initial array analysis (Figure 1B), only miR-455-3p passed the Bonferroni correction $(P \leq 0.05)$, likely due to the marked increase in its expression (12-fold) as well the $P$ value in plasma from patients with sDSP. Of note, miR-455-3p was not elevated in brain tissue from patients with HIV/AIDS with or without sDSP (Asahchop and Power, unpublished observations). However, $\mathrm{miR}-455-3 \mathrm{p}$ was recently reported to be associated with Alzheimer disease in both plasma and brain tissue (37). Moreover, amyloid- $\beta$ exposure to neurons resulted in an induction of miR-455-3p expression levels, similar to the present findings of Vpr exposure to neurons (Supplemental Figure 3). miR-455-3p is also implicated in the regulation of several malignancies, including esophageal, colon, breast, and prostate cancers, albeit with dichotomous effects showing that both promotion and suppression of tumor growth are involved (20, 38-40). These studies underscore the capacity for miR-455-3p to be upregulated and exert biological actions in different systems. There was variation in the individual patient expression of miR-455-3p in both cohorts by $\mathrm{qPCR}$, as shown in Figures 2 and 3, with some samples showing minimal expression. This diversity in findings might be due to factors not apparent from the present studies and warrant further studies. The upregulation of miR-455-3p in both cohorts in our study despite apparent viral suppression implies that there is likely low-level viral replication with accompanying release of $\mathrm{Vpr}$ or local inflammation. Indeed, previous studies have shown detectable levels of $\mathrm{Vpr}(10-32 \mathrm{nM})$ in the cerebrospinal fluid of HIV-1 patients with or without concurrent ART (41).

A key aspect of delineating useful biomarkers is to understand their relationship to the underlying disease process. For miRNAs, an appreciation of their effects on gene regulation and the associated biological consequences is imperative. $\mathrm{miR}-455-3 \mathrm{p}$ has multiple predicted mRNA targets, including $N G F$, which we experimentally validated herein. NGF is a pivotal neurotrophic factor involved in many aspects of peripheral nerve biology, including the development of sDSP (42). In sDSP models from our group, NGF was suppressed in hDRGs, and the sDSP phenotype was reversed by NGF treatment (24). An interesting finding in the present studies was that of expression of NGF in both fetal hDRG neurons and differentiated human neuronal cells. Because NGF synthesis is not described in adult neurons and not generally thought to be synthesized by developing neurons, its immunoreactivity in neurons might reflect uptake of NGF by these cells. Given this possibility, consideration of NGF regulation in other hDRG cell types and associated neuronal uptake warrants consideration. Although the expression of NGF in hDRG neurons remains uncertain, especially in humans, our studies indicated that NGF immunoreactivity was colocalized with $\beta$-III expression, implying that NGF is expressed by hDRG neurons (Figure 4C, inset). Nonetheless, local NGF improved symptoms of neuropathic pain in human clinical trials but did not influence axonal regeneration based on skin biopsy analyses (43). This latter finding does not exclude NGF's potential contribution to nerve restoration in sDSP but suggests that other compounds, such as NGF analogs alone or in combination, might be efficacious. Of note, other genes that are relevant to sDSP were targeted by miR-455-3p, including NTRK2, IGF1, and P75NTR. Although we did not validate the effects of the miR-455-3p mimic on these genes, their dysregulation might also affect neuronal process development that we observed following transduction of hDRGs as well as SK-N-SH neuronal cells. Transfection of miR-455-3p alone or miR-455-3p with an antagomir significantly suppressed or rescued, respectively, NGF and $\beta$-III tubulin expression levels in the SK-N-SH cells (Figure 5). A trend only in NGF reduction was observed in the hDRG cultures with $\mathrm{miR}-455-3 \mathrm{p}$ transfection despite a significant reduction in $\beta$-III tubulin (Figure 4 and Supplemental Figure 4). Overall, these findings highlight 2 points: miR-455-3p might regulate other relevant genes, such as NTRK2 or IGF1, but the efficiency of this miRNA could be influenced by the diversity 
of cell types found in the hDRG cultures, including Schwann cells, neuronal soma, and macrophages, all of which can express $N G F$ and other genes modulated by miR-455-3p. Further studies are warranted to validate the other genes targeted by $\mathrm{miR}-455-3 \mathrm{p}$ in different relevant cell types beyond examining $\beta$-III tubulin and NGF.

The present studies have several limitations. The small number of subjects in each cohort could bias the interpretation of the results, especially the modest effect in the Validation Cohort, but it was reassuring that the HCs, or non-DSP subjects, did not display elevated miR-455-3p expression levels in plasma. Another concern was the small number of miRNAs showing upregulation in the array analyses that could not be amplified subsequently by qPCR; multiple attempts were made to verify the array results for miR-1825 and miR-1281 without success due to unclear technical obstacles, although miR-940 was readily detectable by PCR. Taken together, the results from our study suggest that NGF suppression results from upregulation of $\mathrm{miR}-455-3 \mathrm{p}$ that is induced in sensory neurons by the HIV-1 Vpr. Finally, extension of current findings in a pertinent animal model, such as crossing vpr- or gp120-transgenic animals with an miR-455-3p-deficient mouse, when it becomes available would verify the present observations as well as provide further insights into the mechanisms underlying sDSP.

In summary, induction of $\mathrm{miR}-455-3 \mathrm{p}$ in plasma from HIV/AIDS patients was indicative of the diagnosis of sDSP in 2 independent cohorts. Moreover, miR-455-3p putatively targeted multiple genes that could contribute to sDSP, including NGF. Transfection of human neuronal cultures with miR-455-3p suppressed NGF and $\beta$-III tubulin expression that was also evident as reduced detectable neuronal processes. These findings suggest that miR-455-3p might serve as a potential biomarker for sDSP that could be developed further as an indicator of treatment response in future studies of sDSP and perhaps other sensory neuropathies.

\section{Methods}

Patient cohorts and peripheral neuropathy diagnosis. Study participants included HIV-1-seropositive patients receiving active care at the SAC in Calgary, Alberta, Canada - the Discovery Cohort (sDSP, $n=29$, non-DSP $n=40)(1,44-46)$ - and the St. Vincent's Hospital in Sydney, NSW, Australia - the Validation Cohort ( $\operatorname{sDSP} n=16$, non-DSP $n=20$, and HC $n=15)(47-51)-$ as well as uninfected HCs recruited at each site. The diagnosis of SDSP was based on established criteria, including neurological symptom profile and physical signs on examination (46). The percentage of non-White ethnicities is provided for the Discovery Cohort (Table 1) whereas the Validation Cohort patients were all White. Routine laboratory tests were performed on all patients' samples immediately after diagnosis. Written informed consent (approved by the University of Calgary, E-17256, and St. Vincent's Hospital, HREC/15/SVH/425) was obtained from each subject, and blood samples were collected from each subject at the time of neurological assessment, from which plasma was harvested and stored at $-80^{\circ} \mathrm{C}$.

Experimental design. Mature miRNAs were analyzed by GeneSpring for differential expression in the plasma samples from the Discovery Cohort using the Affymetrix 3.0 array hybridization chips (Affymetrix/ Thermo Fisher Scientific). The miRNAs with the highest fold change and $P$ value less than 0.05 , including those showing a Bonferroni correction $P$ value of less than 0.05 , were selected for further analysis. Machine learning analyses (Random Forest and Support Vector) were applied to the data to confirm and strengthen the analysis. Selected miRNAs from microarray hybridization were validated in selected samples of the Discovery Cohort (sDSP $n=16$, non-DSP $n=16$, and HC $n=9$ ) by qPCR. Using an independent cohort from another group (Validation Cohort), these selected miRNAs were again examined by qPCR.

miRNA extraction from plasma samples, miRNA microarray, and $q P C R$. Using miRNeasy Serum/Plasma Kit (Qiagen), total RNA was extracted from plasma of whole blood collected in EDTA tubes as previously described (44). The Affymetrix 3.0 GeneChip miRNA Array (Affymetrix/Thermo Fisher Scientific) containing human mature miRNA $(n=1,733)$ and pre-miRNA $(n=1,658)$ probes was used for array hybridization as previously described (44). To validate differential expression of miRNAs in the Discovery and Validation Cohorts, a qPCR was performed for specific miRNAs (44). Exiqon miRCURY LNA PCR primer assay (product number 204035 and 204094 for miR-455-3p and miR-940, respectively) and diluted cDNA (1:20) were used for qPCR amplification. miRNA expression data were normalized using Exiqon LNA primer assay $5 \mathrm{~s}$ rRNA (product number 203906) for cellular miRNA or miR-16-5p (product number 205702) for plasma miRNA.

Cell cultures. SK-N-SH cells (ATCC HTB-11) were grown in DMEM supplemented with 10\% FBS, 1\% L-glutamine (Thermo Fisher Scientific), 1\% nonessential amino acids (Thermo Fisher Scientific), 1\% sodium pyruvate (Thermo Fisher Scientific), 1\% dextrose (Thermo Fisher Scientific), and 1\% penicillin and 
streptomycin (Thermo Fisher Scientific). SK-N-SH cells $(10,000$ to 15,000$)$ were plated in 96-well culture plates that were coated with poly-L-lysine for 1 hour and differentiated in complete culture medium containing $1 \mu \mathrm{M}$ dibutyryl cAMP (MilliporeSigma) for 48 hours.

hDRG cultures were prepared from 17- to 19-week fetuses from therapeutic abortions with consent (approved by the University of Alberta Ethics Committee), as described previously (22, 52). The hDRGs were aseptically isolated from fetal spinal cords and placed into a $1.5-\mathrm{ml}$ tube containing DMEM (Thermo Fisher Scientific). The hDRGs were rinsed once and then transferred into another $1.5-\mathrm{ml}$ tube and enzymatically treated with $0.1 \%$ collagenase (MilliporeSigma) for 90 minutes at $37^{\circ} \mathrm{C}$. hDRGs were then mechanically dissociated by trituration by repeated pipetting of cells every 5 minutes using a 1 -ml pipette tip ( 3 times) and then $200-\mu 1$ pipette tip (once). The cell suspension was overlaid with $15 \%$ BSA. The cells were passaged through a 70- $\mu \mathrm{m}$ cell strainer to filter and subsequently centrifuged at $200 \mathrm{~g}$ for 10 minutes. The pellet was resuspended in DMEM supplemented with 10\% FBS (Thermo Fisher Scientific), 1\% L-glutamine (Thermo Fisher Scientific), 1\% nonessential amino acids (Thermo Fisher Scientific), 1\% sodium pyruvate (Thermo Fisher Scientific), 1\% dextrose (Thermo Fisher Scientific), and 1\% penicillin and streptomycin (Thermo Fisher Scientific) and plated in plates coated with poly-L-lysine $(0.01 \%)$.

Exposure to HIV-1 Vpr and miRNA transfection. At 48 hours after differentiation of cultured SK-NSHs, cultures were exposed to HIV-1 Vpr (Kinakeet Biotechnology) for 48 hours. Similarly, differentiated SK-N-SH or cultured hDRG cultures were transfected with an miR-455-3p mimic (GE Healthcare Dharmacon Inc.), an miR-455-3p inhibitor (antagomir) (GE Healthcare Dharmacon Inc.), or an miRNA-NC (GE Healthcare Dharmacon Inc.) using Lipofectamine RNAiMAX (Invitrogen) according to the manufacturer's instructions and as described previously in a concentration-dependent manner for 48 hours (59).

In-cell Western analysis. After exposure to HIV-1 Vpr, SK-N-SH cells were fixed with 4\% paraformaldehyde (PFA), blocked, and incubated overnight with primary antibodies (anti- $\beta$-III tubulin antibody [MilliporeSigma, T8660, 1:200] and anti-NGF antibody [Abcam, ab6199, 1:200]). After overnight incubation, cells were washed in PBS containing $0.1 \%$ Tween 20 and incubated with a donkey anti-mouse IRDye $800 \mathrm{CW}$ (1:800) or donkey anti-rabbit IRDye 800CW (1:800) for 2 hours. After 2 hours, the secondary antibodies were removed, and cells were washed with PBS/0.1\% Tween 20 and left to dry in the dark before quantification of protein expression using the Odyssey CLx Infrared Imaging System (LI-COR Biosciences) (53). The transfections of miR-455-3p, antagomir, and miRNA-NC, in SK-N-SH or hDRGs, were investigated similarly. The immunoreactivity for each host protein was expressed as a percentage of the control (miRNA-NC-transduced) cells.

Immunofluorescence detection. Chamber $\mu$-slides ( 8 wells) (ibidi, Germany) were coated with poly-L-lysine for 1 hour. hDRGs or SK-N-SH cells were then cultured or differentiated, respectively in triplicates or quadruplets, for 48 hours; fixed with 4\% PFA; and blocked with a blocking buffer (LI-COR Biosciences) for 1 hour at room temperature. Primary antibodies (1:200 dilution) specific to $\beta$-III tubulin and NGF were applied to each chamber and incubated at $4^{\circ} \mathrm{C}$ overnight. After overnight incubation, cells were washed with PBS/ 0.1 Tween 20 and incubated with Alexa Fluor secondary antibody, goat anti-mouse (Alexa488), or goat anti-rabbit (Alexa568) (antibodies diluted in blocking buffer (1:800). Cell nuclei were stained by incubating with DAPI for 15 minutes. Images were captured using an Olympus IX-81 confocal microscope with a Yokagawa CSU-10 spinning disk confocal scan head (Quorum Technologies) using Volocity software (54). Total neurite outgrowth length was analyzed and quantified by uploading the captured images into WIS-NeuroMath software (55).

Bioinformatic analysis. GeneSpring version 12.6 (Agilent Technologies) was used to normalize and analyze array data to identify differentially expressed miRNAs in the Discovery Cohort sDSP and nonDSP groups, as previously described, including a Bonferroni correction (44). The array data have been submitted to National Center for Biotechnology Information Gene Expression Omnibus database with accession number GSE118535. Briefly, to identify differentially expressed miRNAs, the median of each probe set in the sDSP or non-DSP patients was calculated and the significance of any differences determined by Mann-Whitney unpaired test on data subjected to GeneSpring normalization. A cutoff fold change $(>2)$ in relative miRNA abundance was applied, and a $P$ value of less than 0.05 was considered statistically significant. This was followed by a correction for multiple comparison (Bonferroni correction). The miRNA targets were predicted by miRwalk, http://zmf.umm.uni-heidelberg.de/apps/zmf/ mirwalk/ (an algorithm for predicted and validated miRNA targets). This algorithm predicts targets based on the probability of seed complementarity to the 3' UTR of the target gene. Predicted target genes were 
further compared with 8 other prediction algorithms (miRDB, DIANA-microT v3.0, TargetScan v6.2, miRanda, PITA, PICTAR5, RNA22, and RNAhybrid).

Statistics. ROC curve analyses were used to predict the diagnosis of sDSP or non-DSP based on the miRNAs obtained from array hybridization and the subsequent qPCR analyses. One-way ANOVA (Kruskal-Wallis) test was used to compare the relative fold change of miRNA expression obtained from qPCR in sDSP versus non-DSP patients, and a $P$ value of less than 0.05 was considered significant. A correlation analysis of selected miRNAs and patients' variables was done by Spearman's correlation coefficient. All statistical analyses were performed using GraphPad Prism (version 6.0). All data are presented as mean \pm SEM. Random Forest and an improved sparse Support Vector Machines analyses were applied to the expression data of 1,733 mature human miRNAs from the array hybridization data and managed to stabilize the results by random subsampling (56-58). More specifically, a stabilized Random Forest approach and stabilized sparse Support Vector Machines with adapt L1 norm were applied with repetition 1,000 and 100 times, respectively. In each run we drew a random subsample of the data, followed by the methods of Random Forest and Support Vector Machines, and a small subset of miRNAs stood out as the potential biomarkers. The selected miRNA by the algorithm was scored 1 (if selected) or 0 (if not selected). After running the algorithm multiple times, the overall frequency of occurrence was used to determine important miRNAs. The top-ranking miRNAs with respect to the overall frequency can be considered the potential biomarkers to separate the sDSP and non-DSP groups. Because a large dimension of the miRNA expression data might cause unstable results to the biomarker selection, alternatively, we screened out approximately two thirds of the candidate miRNAs based on the $P$ values of a 2-tailed sample $t$ test and kept the first 500 miRNAs to apply the aforementioned methods. The 10 top-ranking miRNAs by the methods of Random Forest and sparse Support Vector Machines for the unscreened sDSP data were selected.

Study approval. The present study in human subjects was reviewed and approved by the University of Calgary, Calgary, Alberta, Canada (E-17256), and the St. Vincent's Hospital Sydney, Sydney, Australia (HREC/15/SVH/425). A signed informed consent document was obtained from each participant before inclusion in the study and blood collection.

\section{Author contributions}

ELA and CP designed the experiments and wrote the paper. ELA, WGB, and AK performed all experiments. ELA, PAC, WGB, DY, and LK analyzed results. DWZ advised on experimental approaches and interpretations. BJB, MJG, and CP provided care and collected clinical data for all the patients. All authors read and approved the final manuscript.

\section{Acknowledgments}

These studies were supported by a Canadian Institutes of Health Research (CIHR) Emerging Team grant (to CP and MJG). ELA was supported by fellowships from CIHR and Alberta Innovates - Health Solutions. CP holds a Canada Research Chair in Neurological Infection and Immunity (Tier 1).

Address correspondence to: Christopher Power, 6-11 Heritage Medical Research Centre, University of Alberta, Edmonton, Alberta, Canada. Phone: 780.407.1938; Email: chris.power@ualberta.ca.

1. Vivithanaporn P, et al. Neurologic disease burden in treated HIV/AIDS predicts survival: a population-based study. Neurology. 2010;75(13):1150-1158.

2. Bruce RD, et al. 2017 HIV Medicine Association of Infectious Diseases Society of America Clinical Practice Guideline for the Management of Chronic Pain in Patients Living With Human Immunodeficiency Virus. Clin Infect Dis. 2017;65(10):1601-1606.

3. Navis A, Jiao J, George MC, Simpson D, Robinson-Papp J. Comorbid pain syndromes in HIV-associated peripheral neuropathy. Pain Med. 2018;19(7):1445-1450.

4. Aziz-Donnelly A, Harrison TB. Update of HIV-associated sensory neuropathies. Curr Treat Options Neurol. 2017;19(10):36.

5. Kaku M, Simpson DM. HIV neuropathy. Curr Opin HIV AIDS. 2014;9(6):521-526.

6. van der Donk WA, et al. Inactivation of ribonucleotide reductase by (E)-2'-fluoromethylene-2'-deoxycytidine 5'-diphosphate: a paradigm for nucleotide mechanism-based inhibitors. Biochemistry. 1996;35(25):8381-8391.

7. Benevides MLACSE, Filho SB, Debona R, Bergamaschi ENC, Nunes JC. Prevalence of peripheral neuropathy and associated factors in HIV-infected patients. J Neurol Sci. 2017;375:316-320.

8. Arenas-Pinto A, et al. Peripheral neuropathy in HIV patients in sub-Saharan Africa failing first-line therapy and the response to second-line ART in the EARNEST trial. J Neurovirol. 2016;22(1):104-113.

9. Hahn K, et al. A placebo-controlled trial of gabapentin for painful HIV-associated sensory neuropathies. $J$ Neurol. 
2004;251(10):1260-1266.

10. Evans SR, et al. Simplification of the research diagnosis of HIV-associated sensory neuropathy. HIV Clin Trials. 2008;9(6):434-439.

11. Abrams DI, et al. Cannabis in painful HIV-associated sensory neuropathy: a randomized placebo-controlled trial. Neurology. 2007;68(7):515-521.

12. Dimitrova A, Murchison C, Oken B. Acupuncture for the treatment of peripheral neuropathy: a systematic review and meta-analysis. J Altern Complement Med. 2017;23(3):164-179.

13. Szczudlik A, et al. Diagnosis and management of neuropathic pain: review of literature and recommendations of the Polish Association for the Study of Pain and the Polish Neurological Society - Part Two. Neurol Neurochir Pol. 2014;48(6):423-435.

14. McArthur JC, et al. A phase II trial of nerve growth factor for sensory neuropathy associated with HIV infection. AIDS Clinical Trials Group Team 291. Neurology. 2000;54(5):1080-1088.

15. Mangus LM, et al. Unraveling the pathogenesis of HIV peripheral neuropathy: insights from a simian immunodeficiency virus macaque model. ILAR J. 2014;54(3):296-303.

16. Yeung H, Krentz HB, Gill MJ, Power C. Neuropsychiatric disorders in HIV infection: impact of diagnosis on economic costs of care. AIDS. 2006;20(16):2005-2009.

17. Makarova JA, et al. Intracellular and extracellular microRNA: An update on localization and biological role. Prog Histochem Cytochem. 2016;51(3-4):33-49.

18. Fabbri M. MicroRNAs and miRceptors: a new mechanism of action for intercellular communication Philos Trans $R$ Soc Lond, B, Biol Sci. 2018;373(1737)

19. Ghibaudi M, Boido M, Vercelli A. Functional integration of complex miRNA networks in central and peripheral lesion and axonal regeneration. Prog Neurobiol. 2017;158:69-93.

20. Liu A, et al. Antagonizing miR-455-3p inhibits chemoresistance and aggressiveness in esophageal squamous cell carcinoma. Mol Cancer. 2017;16(1):106.

21. Lodge R, et al. Host microRNAs-221 and -222 inhibit HIV-1 entry in macrophages by targeting the CD4 viral receptor. Cell Rep. 2017;21(1):141-153

22. Acharjee S, et al. HIV-1 viral protein R causes peripheral nervous system injury associated with in vivo neuropathic pain. FASEB J. 2010;24(11):4343-4353.

23. Cheng $C$, et al. Evidence for epigenetic regulation of gene expression and function in chronic experimental diabetic neuropathy. J Neuropathol Exp Neurol. 2015;74(8):804-817.

24. Webber CA, et al. Nerve growth factor acts through the TrkA receptor to protect sensory neurons from the damaging effects of the HIV-1 viral protein, Vpr. Neuroscience. 2013;252:512-525.

25. Chen H, et al. Peripheral neuropathy in ART-experienced patients: prevalence and risk factors. J Neurovirol. 2013;19(6):557-564

26. Evans SR, et al. Peripheral neuropathy in HIV: prevalence and risk factors. AIDS. 2011;25(7):919-928.

27. Holzinger ER, et al. Mitochondrial DNA variation and HIV-associated sensory neuropathy in CHARTER. J Neurovirol. 2012;18(6):511-520.

28. Lopate G, Pestronk A, Evans S, Li L, Clifford D. Anti-sulfatide antibodies in HIV-infected individuals with sensory neuropathy. Neurology. 2005;64(9):1632-1634.

29. Li X, et al. Interruption of KLF5 acetylation converts its function from tumor suppressor to tumor promoter in prostate cancer cells. Int J Cancer. 2015;136(3):536-546.

30. Zhou L, et al. Correlates of epidermal nerve fiber densities in HIV-associated distal sensory polyneuropathy. Neurology. 2007;68(24):2113-2119.

31. Tsuda M, Beggs S, Salter MW, Inoue K. Microglia and intractable chronic pain. Glia. 2013;61(1):55-61.

32. Tyor WR, et al. Cytokine expression of macrophages in HIV-1-associated vacuolar myelopathy. Neurology. 1993;43(5):1002-1009.

33. Ellis RJ, et al. Continued high prevalence and adverse clinical impact of human immunodeficiency virus-associated sensory neuropathy in the era of combination antiretroviral therapy: the CHARTER Study. Arch Neurol. 2010;67(5):552-558.

34. Stavros K, Simpson DM. Understanding the etiology and management of HIV-associated peripheral neuropathy. Curr HIV/ AIDS Rep. 2014;11(3):195-201.

35. Polydefkis M. Skin biopsy findings predict development of symptomatic neuropathy in patients with HIV. Nat Clin Pract Neurol. 2006;2(12):650-651.

36. Lakritz JR, et al. Monocyte traffic, dorsal root ganglion histopathology, and loss of intraepidermal nerve fiber density in SIV peripheral neuropathy. Am J Pathol. 2015;185(7):1912-1923.

37. Kumar S, Vijayan M, Reddy PH. MicroRNA-455-3p as a potential peripheral biomarker for Alzheimer's disease. Hum Mol Genet. 2017;26(19):3808-3822.

38. Zhao Y, et al. MicroRNA-455-3p functions as a tumor suppressor by targeting eIF4E in prostate cancer. Oncol Rep. 2017;37(4):2449-2458.

39. Li Z, et al. MicroRNA-455-3p promotes invasion and migration in triple negative breast cancer by targeting tumor suppressor EI24. Oncotarget. 2017;8(12):19455-19466.

40. Zheng J, Lin Z, Zhang L, Chen H. MicroRNA-455-3p inhibits tumor cell proliferation and induces apoptosis in HCT116 human colon cancer cells. Med Sci Monit. 2016;22:4431-4437.

41. Mamik MK, et al. HIV-1 viral protein R activates NLRP3 inflammasome in microglia: implications for HIV-1 associated neuroinflammation. J Neuroimmune Pharmacol. 2017;12(2):233-248.

42. Rask CA. Biological actions of nerve growth factor in the peripheral nervous system. Eur Neurol. 1999;41(suppl 1):14-19.

43. Schifitto G, et al. Long-term treatment with recombinant nerve growth factor for HIV-associated sensory neuropathy. Neurology. 2001;57(7):1313-1316

44. Asahchop EL, Akinwumi SM, Branton WG, Fujiwara E, Gill MJ, Power C. Plasma microRNA profiling predicts HIV-associated neurocognitive disorder. AIDS. 2016;30(13):2021-2031.

45. McCombe JA, Vivithanaporn P, Gill MJ, Power C. Predictors of symptomatic HIV-associated neurocognitive disorders in universal health care. HIV Med. 2013;14(2):99-107. 
46. Pettersen JA, et al. Sensory neuropathy in human immunodeficiency virus/acquired immunodeficiency syndrome patients: protease inhibitor-mediated neurotoxicity. Ann Neurol. 2006;59(5):816-824.

47. Cysique LA, et al. Erratum to: White matter measures are near normal in controlled HIV infection except in those with cognitive impairment and longer HIV duration. J Neurovirol. 2017;23(4):548-549.

48. Bloch $\mathrm{M}$, et al. A screening strategy for HIV-associated neurocognitive disorders that accurately identifies patients requiring neurological review. Clin Infect Dis. 2016;63(5):687-693.

49. Gates TM, Cysique LA, Siefried KJ, Chaganti J, Moffat KJ, Brew BJ. Maraviroc-intensified combined antiretroviral therapy improves cognition in virally suppressed HIV-associated neurocognitive disorder. AIDS. 2016;30(4):591-600.

50. Cysique LA, Maruff P, Brew BJ. Variable benefit in neuropsychological function in HIV-infected HAART-treated patients. Neurology. 2006;66(9):1447-1450.

51. Brew BJ. The peripheral nerve complications of human immunodeficiency virus (HIV) infection. Muscle Nerve. 2003;28(5):542-552.

52. Acharjee S, et al. Proteinase-activated receptor-1 mediates dorsal root ganglion neuronal degeneration in HIV/AIDS. Brain. 2011;134(Pt 11):3209-3221.

53. Mamik MK, et al. Insulin treatment prevents neuroinflammation and neuronal injury with restored neurobehavioral function in models of HIV/AIDS neurodegeneration. J Neurosci. 2016;36(41):10683-10695.

54. Asahchop EL, et al. Reduced antiretroviral drug efficacy and concentration in HIV-infected microglia contributes to viral persistence in brain. Retrovirology. 2017;14(1):47.

55. Rishal I, et al. WIS-NeuroMath enables versatile high throughput analyses of neuronal processes. Dev Neurobiol. 2013;73(3):247-256.

56. Guyon I, Weston J, Barnhill S, Vapnik V. Gene selection for cancer classification using support vector machines. Mach Learn. 2002;46(1-3):389-422.

57. Huang L, Zhang HH, Zeng ZB, Bushel PR. Improved sparse multi-class SVM and its application for gene selection in cancer classification. Cancer Inform. 2013;12:143-153.

58. Zhang HH, Ahn J, Lin X, Park C. Gene selection using support vector machines with non-convex penalty. Bioinformatics. 2006;22(1):88-95

59. Xu Z, et al. MicroRNAs upregulated during HIV infection target peroxisome biogenesis factors: Implications for virus biology, disease mechanisms and neuropathology. PLOS Pathogen. 2017;13(6):e1006360. 